\title{
Sarcoma primário da artéria pulmonar - um caso clínico
}

\author{
Primary Pulmonary Artery Sarcoma - case report \\ Heloísa Ribeiro, Luísa Moreira, Fernando Moreira
Serviço de Medicina Interna, Centro Hospitalar de Entre o Douro e Vouga, Santa Maria da Feira, Portugal
}

\section{Resumo}

0 sarcoma da artéria pulmonar é uma neoplasia muito rara, que se manifesta de forma inespecífica. Descreve-se o caso de um indivíduo do sexo masculino, de 88 anos, com uma massa na artéria pulmonar, de $6,5 \mathrm{~cm}$ de maior eixo, condicionando oclusão de cerca de 80\%. 0 doente foi submetido a exérese da massa, com histologia compatível com Angiossarcoma epitelióide/ Leiomiossarcoma, e a radioterapia. A cirurgia constitui o tratamento recomendado, mesmo quando não há intenção curativa.

Palavras-chave. Sarcoma, Artéria Pulmonar, Tumor vascular.

\section{Introdução}

0 Sarcoma da Artéria Pulmonar (SAP) é um tumor muito raro. Desde que foi descrito pela primeira vez em 1923 por Mandelstamm, cerca de 250 casos foram apresentados na literatura internacional, muitos dos quais diagnosticados pós-mortem ${ }^{1} .0$ prognóstico associado a este tipo de tumores é reservado, estando recomendada a ressecção cirúrgica, mesmo na ausência de intenção curativa ${ }^{1,2}$.

Descreve-se 0 caso de um paciente com sarcoma da artéria pulmonar.

\section{Caso clínico}

Descreve-se o caso de um doente do sexo masculino, de 88 anos, previamente autónomo nas actividades da vida diária. Apresentava antecedentes de traumatismo craneo-encefálico em 1987 após queda de bicicleta, Acidente Isquémico Transitório (AIT) em 2000, bócio multinodular submetido a tiroidectomia total em 2002 e hipertensão arterial, ex-fumador desde há 30 anos (15 unidades maço ano), consumo diário de álcool quantificado em 12g, habitualmente medicado com Levotiroxina $0.1 \mathrm{mg} / \mathrm{dia}$, Perindopril $4 \mathrm{mg} /$ dia, Furosemida 40mg/dia.

Recorreu ao Serviço de Emergência (SE) a 05.02.2013 por mal-estar inespecífico e afasia global, com recuperação total em menos de 24 horas. Realizou tomografia computadorizada (TC) craneo-encefálica (CE), onde se constatou lacuna lenticular posterior no hemisfério direito, atrofia cerebral focal cortical temporo-basal esquerda e atrofia cerebral difusa cortico-subcortical de grau ligeiro. Concluiu-se por AIT provável, tendo tido alta orientado para Consulta Externa de Neurologia.

A 17.02 o doente foi novamente conduzido ao SE por prostração. No dia anterior tinha sido encontrado caído, agitado e confuso, tendo recuperado e recusado ida ao SE. Negava dor torácica, dispneia, febre, mordedura de língua ou perda de continência de esfíncteres. Ao exame objectivo apresentava-se corado e hidratado, tensão arterial: 128/80 mmHg, frequência cardíaca: 96 bat/ min, saturação de 02

\begin{abstract}
Pulmonary artery sarcoma is a very rare tumour, which presents with non-specific symptoms. We present a case of an 88 years old man with a pulmonary artery tumour of 6.5 $\mathrm{cm}$ which occluded $80 \%$. Patient was submitted to surgery, the histology showed Angiosarcoma/Leiomyosarcoma, and radiotherapy. Surgery is recommended even when there is no curative intention.
\end{abstract}

Keywords. Sarcoma, Pulmonary Artery, Vascular tumour.
100\% (em ar ambiente). Auscultação cardíaca rítmica, com sopro sistólico grau II/VI. Auscultação pulmonar com murmúrio vesicular mantido e simétrico, com crepitações audíveis em ambas as bases. Apresentava edema bimaleolar. Ao exame neurológico apresentava-se vigil, pupilas isocóricas, isofotorreactivas. Oculomotricidade mantida. Discurso fluente, sem disartria. Mimica facial mantida, sem paresia. Restantes nervos cranianos sem alterações. Força muscular preservada nos membros superiores e inferiores. Reflexo cutâneo plantar em flexão bilateralmente. Reflexos osteotendinosos simétricos (2+).

$\mathrm{Na}$ investigação efectuada constatou-se electrocardiograma em ritmo sinusal com bloqueio completo de ramo direito e hemibloqueio anterior esquerdo e gasimetria em ar ambiente com discreta alcalose respiratória $(\mathrm{pH} 7.5, \mathrm{pCO} 234 \mathrm{mmHg}, \mathrm{p02} 70 \mathrm{mmHg}$, $\mathrm{Na}+140 \mathrm{mmol} / \mathrm{L}, \mathrm{K}+3.2 \mathrm{mmol} / \mathrm{L}$, Lactatos $1.1 \mathrm{mmol} / \mathrm{L}, \mathrm{HCO}-$ $27.1 \mathrm{mmol} / \mathrm{L}, \mathrm{BE} 4.2 \mathrm{mmo} / \mathrm{L}$, saturação de 02 95\%). Analiticamente salientava-se Proteína C Reactiva $48.6 \mathrm{mg} / \mathrm{L}$, sem alterações do hemograma, ionograma e função renal. Realizou TC-CE que revelou hemorragia subdural subaguda e subaracnoideia, pelo que efetuou Angio-TC, que excluiu malformações aneurismáticas. Neste contexto foi considerada a hipótese da perda de consciência ter sido anterior à queda. Realizou ecocardiograma transtorácico que demonstrou dilatação moderada a grave das cavidades direitas; insuficiência tricúspide moderada a grave, permitindo estimar pressão sistólica da artéria pulmonar de $80 \mathrm{mmHg}$; veia cava inferior dilatada sem variabilidade inspiratória e disfunção grave do ventrículo direito. Na sequência das alterações detectadas, foi solicitado TC torácico, onde se observou volumosa lesão endoluminal na artéria pulmonar, desde a válvula pulmonar até à bifurcação do tronco e porções proximais dos ramos respetivos, medindo $6.5 \mathrm{~cm}$ de maior eixo, condicionando oclusão luminal de 80\% (Figura 1). 0 doente foi orientado para 0 Serviço de Cirurgia Cardiotorácica tendo-se procedido a exérese da massa e anuloplastia da tricúspide. 0 exame histológico foi compatível com Angiossarcoma epitelióide/Leiomiossarcoma com "marcação aberrante" para marcadores vasculares e citoqueratinas. 0 pós-operatório foi complicado por Pneumonia 
Figura 1. Lesão endoluminal na artéria pulmonar, localizada desde a válvula pulmonar até à bifurcação do tronco e porções proximais dos ramos respetivos.

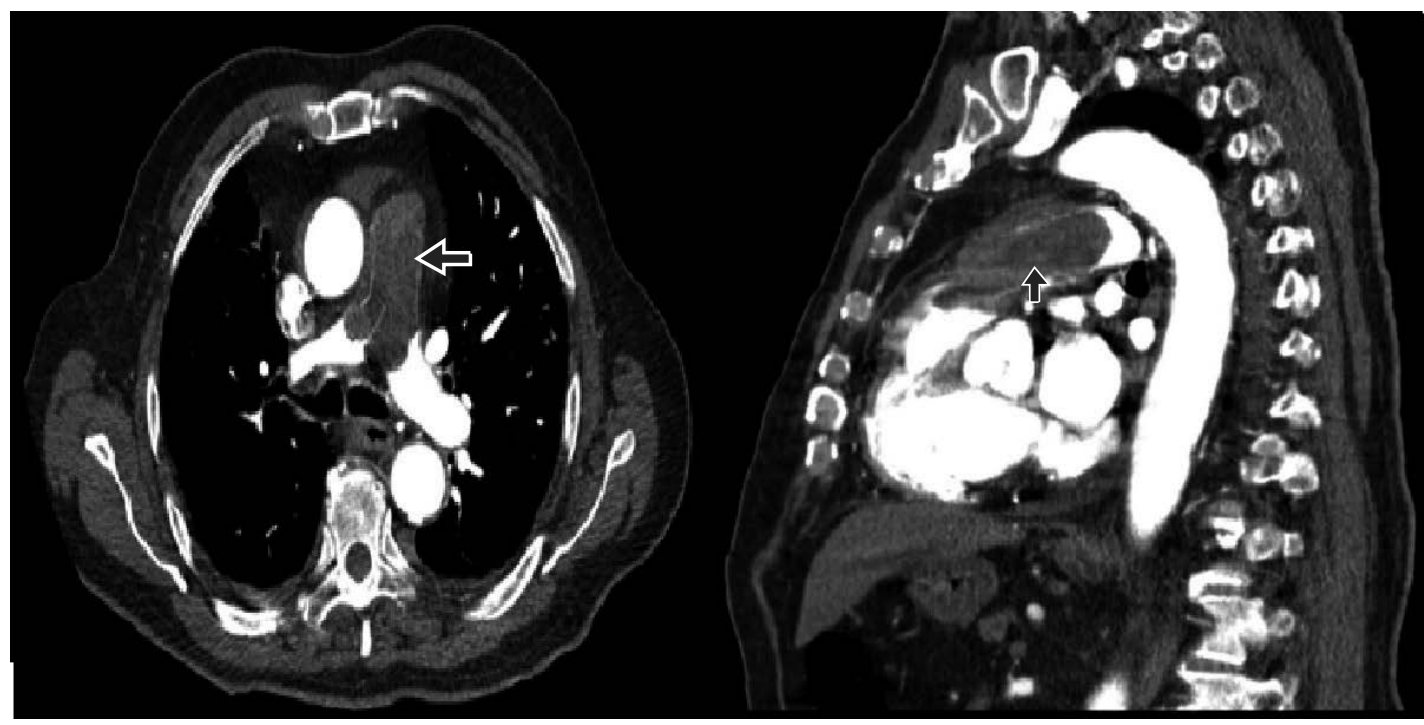

associada aos cuidados de saúde, tendo cumprido antibioterapia com Meropenem $1 \mathrm{gr}$ de 8/8h e Amicacina $1 \mathrm{gr}$ de 24/24h com boa evolução clínica e analítica. À data de alta 0 doente foi orientado para Consulta de Grupo de Oncologia, tendo sido posteriormente submetido a radioterapia. Apresentava razoável estado geral seis meses após o diagnóstico.

\section{Discussão}

O SAP é um tumor raro, cuja idade de diagnóstico varia entre os 13 e os 89 anos, sendo mais frequente nos indivíduos de meia-idade e do género feminino ${ }^{3}$. Histologicamente podem ser classificados em diversos subtipos como rabdomiossarcoma, angiossarcoma, leiomiossarcoma, entre outros. A origem deste tipo de tumor ainda não está claramente definida, apontando-se para a possibilidade de ter proveniência de células mesenquimais da íntima dos vasos ${ }^{4}$, ou da média no caso do leiomiossarcoma ${ }^{1}$, assumindo posteriormente diversos tipos morfológicos.

0 quadro clínico é inespecífico, podendo apresentar-se com tosse, dor torácica, dispneia, hemoptises e síncope ${ }^{5}$, o que torna difícil o diagnóstico diferencial com outras entidades como o tromboembolismo pulmonar agudo e crónico, neoplasia do pulmão e hipertensão pulmonar².

Analiticamente poderá estar presente anemia e velocidade de sedimentação eritrocitária aumentada ${ }^{6}$. No electrocardiograma, bloqueio de ramo direito, hipertrofia ventricular direita, alterações inespecíficas do segmento ST e inversão da onda T. 0 tumor pode ser detetado por ecocardiograma (principalmente transesofágico), tomografia computadorizada, ressonância magnética e modalidades invasivas (como angiografia pulmonar e cateterismo cardíaco)3,6.

No que diz respeito ao tratamento, encontram-se descritos casos abordados de diversas formas, nomeadamente com cirurgia, radioterapia e quimioterapia, em monoterapia ou de forma combinada. Contudo, a resseção cirúrgica é a opção recomendada, mesmo quando não há intenção curativa, uma vez que está descrito que prolonga a sobrevida. Segundo Blackmond et al, deverá ser considerada a possibilidade de quimioterapia neoadjuvante ou adjuvante, esta última na ausência de condições que permitam suportar o tratamento neoadjuvante ${ }^{4}$.

0 prognóstico é reservado, sendo a sobrevida média de 1.5 meses nos doentes não submetidos a cirurgia e de 12 meses naqueles submetidos àquele tratamento ${ }^{3,6}$.

\section{Conclusão}

O Sarcoma da artéria pulmonar é uma entidade rara, estando implícita a dificuldade do diagnóstico (o que não se verificou no caso apresentado dadas as características imagiológicas da massa) e do tratamento. A individualização terapêutica é dificultada pela limitada experiência da sua abordagem, decorrente do reduzido número de casos descritos, da sua distribuição geográfica bem como das características dos doentes descritos na literatura.

\section{Bibliografia}

1. Huo L, Moran CA, Fuller GN, Gladish G, Suster S. Pulmonary Artery Sarcoma - A Clinicopathologic and Immunohistochemical Study of 12 Cases. Am J Clin Pathol 2006; 125:419-424.

2. Adeli SH, Nemati B, Jandaghi M, Riahi MM, Hosseinzadeh F, Salarvand F. Pulmonary Hypertension due to a Pulmonary Artery Leiomyosarcoma: A Case Report. Case Rep Pulmonol. 2013;2013:160619. doi: 10.1155/2013/160619. Epub 2013 Mar 31.

3. Alsoufi B, Slater M, Smith PP, Karamlou T, Mansoor A, Ravichandran P. Pulmonary Artery Sarcoma Mimicking Massive Pulmonary Embolus: A Case Report. Asian Cardiovasc Thorac Ann 2006;14:71-73.

4. Blackmon SH, Rice DC, Correa AM, Mehran R, Putnam JB, Smythe ER, et al. Management of Primary Pulmonary Artery Sarcomas. Ann Thorac Surg 2009; 87:977984.

5. Nakamuraa $Y$, Shimizub T, Fukumotob Y, Sugimurab K, Itoc S, Fujishimaa F, et al. A Case of Angiosarcoma Arising in Trunk of the Right Pulmonary Artery Clinically simulating Pulmonary Thromboembolism. World J Oncol 2012; 3(3):119-123.

6. Neves S, San José J, Vaz Velho H, Oliveira M, Esteves I, Torres S, Magalhães AM. Sarcoma da Artéria Pulmonar — dificuldades diagnósticas e terapêuticas. Rev Port Pneumol 2003; IX (1): 41-51. 